\title{
Abnormal White Matter Integrity in Chronic Users of Codeine-Containing Cough Syrups: A Tract-Based Spatial Statistics Study
}

\begin{abstract}
BACKGROUND AND PURPOSE: Codeine-containing cough syrups have become one of the most popular drugs of abuse in young people in the world. Chronic codeine-containing cough syrup abuse is related to impairments in a broad range of cognitive functions. However, the potential brain white matter impairment caused by chronic codeine-containing cough syrup abuse has not been reported previously. Our aim was to investigate abnormalities in the microstructure of brain white matter in chronic users of codeine-containing syrups and to determine whether these WM abnormalities are related to the duration of the use these syrups and clinical impulsivity.
\end{abstract}

MATERIALS AND METHODS: Thirty chronic codeine-containing syrup users and 30 matched controls were evaluated. Diffusion tensor imaging was performed by using a single-shot spin-echo-planar sequence. Whole-brain voxelwise analysis of fractional anisotropy was performed by using tract-based spatial statistics to localize abnormal WM regions. The Barratt Impulsiveness Scale 11 was surveyed to assess participants' impulsivity. Volume-of-interest analysis was used to detect changes of diffusivity indices in regions with fractional anisotropy abnormalities. Abnormal fractional anisotropy was extracted and correlated with clinical impulsivity and the duration of codeine-containing syrup use.

RESULTS: Chronic codeine-containing syrup users had significantly lower fractional anisotropy in the inferior fronto-occipital fasciculus of the bilateral temporo-occipital regions, right frontal region, and the right corona radiata WM than controls. There were significant negative correlations among fractional anisotropy values of the right frontal region of the inferior fronto-occipital fasciculus and the right superior corona radiata WM and Barratt Impulsiveness Scale total scores, and between the right frontal region of the inferior frontooccipital fasciculus and nonplan impulsivity scores in chronic codeine-containing syrup users. There was also a significant negative correlation between fractional anisotropy values of the right frontal region of the inferior fronto-occipital fasciculus and the duration of codeine-containing syrup use in chronic users.

CONCLUSIONS: Chronic codeine-containing syrup abuse may be associated with disruptions in brain WM integrity. These WM microstructural deficits may be linked to higher impulsivity in chronic codeine-containing syrup users.

ABBREVIATIONS: BIS = Barratt Impulsiveness Scale; $\mathrm{CCS}=$ codeine-containing syrup; FA $=$ fractional anisotropy; IFO $=$ inferior fronto-occipital fasciculus; $\lambda_{1}=$ axial diffusivity; $\lambda_{\perp}=$ radial diffusivity; TBSS $=$ tract-based spatial statistics

C odeine is by far the most widely used opiate in the world. It has been frequently used as an antitussive agent. ${ }^{1}$ It has relatively specific central inhibition of the cough center in the medulla and does not cause respiratory depression. Although codeine is a less addictive

Received December 13, 2013; accepted after revision June 15, 2014

From the Department of Medical Imaging (Y.-w.Q., H.-h.S., G.-h.J.), Guangdong No. 2 Provincial People's Hospital, Guangzhou, China; Department of Medical Imaging (Y.-w.Q.), The First Affiliated Hospital of Gannan Medical University, Ganzhou, China; and Departments of Medical Imaging and Interventional Radiology (X.-f.L.), Cancer Center, Sun Yat-Sen University, Guangzhou, China.

This work was supported by a grant from the Natural Scientific Foundation of China (Grant No. 81201084 for Ying-wei Qiu). The funder had no role in study design, data collection and analysis, decision to publish, or preparation of the manuscript.

Please address correspondence to Ying-wei Qiu, MD, Department of Medical imaging, Guangdong No. 2 Provincial People's Hospital, 510317, Guangzhou, China; e-mail: qiuyw1201@gmail.com opiate than heroin, continued use can result in physical and psychological dependence. ${ }^{2}$ Codeine-containing cough syrups (CCSs) have become one of the most popular drugs of abuse in young people in the world. ${ }^{3}$ The exact neurobiologic mechanism underlying CCS addiction is not fully understood. Codeine dependence is different from that of other illicit opioid drugs, and its misuse is associated with a different clinical course. ${ }^{4} \mathrm{CCS}$ contains a combination of codeine, a sympathomimetic, and an antihistamine, all of which have central nervous system action, which is not typical of other opioids. ${ }^{4}$

Understanding the mechanism of CCS dependence and developing better therapies to stop CCS abuse are important because of the increasing incidence of dependency. Emerging technologies

- Indicates open access to non-subscribers at www.ajnr.org

http://dx.doi.org/10.3174/ajnr.A4070 
such as neuroimaging have contributed to new insights into the neuropharmacology of CCS addiction. ${ }^{5-7}$ Hou et $\mathrm{al}^{5}$ used singlephoton emission CT to demonstrate that the availability of dopamine transporter in the striatum and the volume, weight, and technetium Tc99m-TRODAT-1 corpus striatum/whole-brain uptake ratio were significantly less pronounced in CCS users than in controls. Using high-resolution structural MR imaging and resting-state functional MR imaging, our previous studies reported that the gray matter volume of the ventral medial prefrontal cortex and its functional integrity were deficient in chronic CCS users. ${ }^{6,7}$ However, to the best of our knowledge, the integrity of white matter in CCS users has not been investigated. Knowledge of white matter brain abnormalities and any association between these abnormalities and cognitive function in CCS users might facilitate identification of possible pharmacotherapies that could be used to treat this disorder.

Diffusion tensor imaging is an MR imaging technique used to quantitatively investigate white matter axonal integrity in vivo. DTI is based on the measurement of water molecular motion. Axonal structure restricts water diffusion so that it is greater in the axis parallel to the main axis of axons. Fractional anisotropy (FA) is a measure of the degree to which water diffusion is constrained in the brain, and it is widely used as a general index of axonal integrity. ${ }^{8,9}$ The component measures from which FA is derived, the first $\left(\lambda_{1}\right)$ and second $\left(\lambda_{\perp}\right)$ principal eigenvalues, indicate diffusion axial (parallel) and radial (perpendicular) to the primary axis of the axon. These values provide additional insight into the nature of white matter deficits. ${ }^{10,11}$ The former provides an index of axonal injury, and the latter is sensitive to changes in myelination. ${ }^{10,12}$

In this study, DTI was used to investigate the white matter integrity of chronic CCS users. An observer-independent tractbased spatial statistics (TBSS) analysis was performed on the DTI data. This method retains the strengths of voxel-based analysis while addressing some of its drawbacks. Drawbacks include aligning images from multiple subjects and the arbitrariness of the choice of spatial smoothing. ${ }^{13}$ The aims of this study were the following: 1) to investigate differences in the topographic distribution of the integrity of white matter between CCS users and healthy controls, 2) to determine whether there was any relationship among abnormalities in the integrity of white matter and the duration of CCS use and a clinical impulsivity trait in CCS users.

\section{MATERIALS AND METHODS \\ Subjects}

This prospective study was approved by the local ethics committee. Written informed consent was obtained from all subjects. Sixty subjects, including 30 control subjects and 30 chronic CCS users, participated in this study. The CCS users were randomly selected from among patients seeking treatment at the Addiction Medicine Division of Guangdong No.2 Provincial People's Hospital. All the CCS users were screened by using the Diagnostic and Statistical Manual of Mental Disorders-IV criteria. A urine test and an interview were conducted by a clinical psychologist. All patients were naive for other types of illicit drug use. None of the 60 subjects (30 CCS users and 30 controls) included in present study meet the criteria of Diagnostic and Statistical Manual of Mental Disorders-IV for alcohol dependence, and none used alcohol reg- ularly. Patients regularly used cigarettes and denied any use of psychotropic agents in the month before MR imaging. Inclusion criteria for the control subjects were a lack of a diagnosis of substance abuse or dependence. Exclusion criteria for all participants included neurologic illness, schizophrenia, bipolar disorder, prior significant head trauma, positive HIV status, diabetes, hepatitis C, other major medical illness, and left-handedness.

\section{Behavioral Assessments}

The Barratt Impulsiveness Scale (BIS) 11 was surveyed soon after MR imaging to assess participants' impulsivity. The BIS 11 is one of the oldest and most widely used self-reporting measures of impulsive personality traits. This 30 -item self-rated scale evaluates 3 factors: attentional/cognitive traits, which refer to tolerance for cognitive complexity and persistence; motor, which refers to the tendency to act on the spur of the moment; and nonplan impulsivity, which refers to the lack of a sense of the future. Items were rated from 1 (rarely/never) to 4 (almost always/always). All items were summed to determine overall impulsiveness scores. Higher scores indicated greater impulsivity. ${ }^{14}$ The BIS 11 is a valid and reliable instrument for evaluating healthy and psychiatric Chinese populations. ${ }^{15}$

\section{Image Acquisition}

MR imaging data were obtained with a 1.5T MR scanner (Achieva Nova-Dual; Philips, Best, the Netherlands). Each subject lay supine with his or her head snugly fixed by a belt and foam pads. Before the DTI scan, a T1-weighted 3D sequence and fluid-attenuated inversion recovery images were captured to exclude subjects with abnormalities. Diffusion imaging data were acquired in 32 diffusion gradient directions $\left(b=800 \mathrm{~s} / \mathrm{mm}^{2}\right.$ along 32 noncollinear directions) plus $b=0$ reference images by using a singleshot spin-echo-planar sequence to collect diffusion-weighted images. Other imaging parameters were as follows: $\mathrm{TR}=10,793 \mathrm{~ms}$, $\mathrm{TE}=62 \mathrm{~ms}, \mathrm{FOV}=230 \times 230 \mathrm{~mm}^{2}$, matrix $=128 \times 128$, section thickness $=2 \mathrm{~mm}$ with no section gap, and voxel size $=2 \times 2 \times$ $2 \mathrm{~mm}^{3}$.

\section{Data Preprocessing}

All DTI datasets were preprocessed by using the fMRI of the Brain Software Library, Version 4.1.7 (FSL; http://www.fmrib.ox.ac. $\mathrm{uk} / \mathrm{fsl}$ ). The raw DTI dataset was corrected for eddy current distortion and head motion by registering the diffusion-weighted images with the null image. This was performed by using affine transformations in the Diffusion Toolbox, Version 2.0, (part of FSL). ${ }^{16}$ The DTI datasets were then skull-stripped by using the Brain Extraction Tool, Version 1 (part of FSL) to remove background noise and nontissue components. ${ }^{17}$ The diffusion tensor was then calculated by using the DTIFit (from FSL) program for whole-brain volumes to produce FA and axial and radial diffusivities.

\section{TBSS Analysis}

Whole-brain voxelwise statistical analysis of the FA data was performed by using TBSS. ${ }^{13,16}$ The TBSS method minimizes potential misalignment problems of other voxel-based whole-brain forms of analysis by identifying a white-matter "skeleton" restricted only to the center of the major white-matter tracts. FA 
Table 1: Demographic and clinical characteristics of the chronic codeine-containing cough syrup users and controls ${ }^{a}$

\begin{tabular}{|c|c|c|c|c|}
\hline Characteristic & CCS Users $(n=30)$ & Controls $(n=30)$ & Z Value & $P$ Value \\
\hline Age (yr) (mean, range) & $25.07(3.084,20-31)$ & $23.97(2.470,20-29)$ & 2.706 & .133 \\
\hline Sex (male/female) & $28: 2$ & $28: 2$ & - & 1 \\
\hline Education (yr) & $13.03(2.760)$ & $12.07(3.423)$ & 1.126 & .233 \\
\hline Nicotine (No. cigarette/day) & $16.53(9.609)$ & $13.50(8.823)$ & 0.036 & .208 \\
\hline Cough syrup use (yr) (range) & $5.08(1 \sim 8)$ & N/A & - & - \\
\hline Age of first use of cough syrup (yr) (range) & $19.93(12 \sim 30)$ & $\mathrm{N} / \mathrm{A}$ & - & - \\
\hline Mean dose (mL/day) (range) & $487.33(60 \sim 1800)$ & N/A & - & - \\
\hline Total BIS scores & $71.63(4.597)$ & $57.13(5.178)$ & 0.778 & $.000^{\mathrm{b}}$ \\
\hline Attentional impulsivity & $18.27(2.947)$ & $15.57(1.633)$ & 5.341 & $.000^{\mathrm{b}}$ \\
\hline Motor impulsivity & $30.50(2.739)$ & $22.13(3.381)$ & 2.727 & $.000^{\mathrm{b}}$ \\
\hline Nonplan impulsivity & $22.87(1.961)$ & $19.63(2.710)$ & 1.755 & $.000^{\mathrm{b}}$ \\
\hline
\end{tabular}

Note:-NA indicates not applicable; -, no value.

${ }^{a}$ Unless otherwise indicated, data are means \pm SDs.

${ }^{\mathrm{b}} \mathrm{p}<.05$.

values were mapped for each individual onto this standard skeleton for group comparison.

All subjects' FA data were aligned into a common space by using the FSL Nonlinear Registration Tool, FNIRT (http://fsl.fmrib.ox.ac.uk/fsl/ fslwiki/FNIRT), which uses a b-spline representation of the registration warp field. ${ }^{18}$ The cross-subject mean FA image was calculated and used to generate a mean FA skeleton representing the centers of all tracts common to the whole study group. The threshold of the mean FA skeleton was set at 0.2 to exclude voxels that contained gray matter or CSF. Previous studies have also used the same threshold for the FA skeleton. ${ }^{19}$ Each individual subject's aligned FA data were projected onto this masked skeleton by filling the skeleton with FA values from the nearest relevant tract center. The skeletonized FA data were fed into the following voxelwise cross-subject statistics, which were based on a nonparametric permutation test (Randomize, part of FSL; http://fsl.fmrib. ox.ac.uk/fsl/fslwiki/Randomise/UserGuide).

Nonparametric 2-sample independent $t$ tests were used to identify group differences in FA. This comparison was based on a permutation method because of the substantial non-Gaussian distribution of the FA data. ${ }^{13,20}$ Age, years of formal education, and histories of smoking were demeaned and served as nuisance covariates to ensure that any observed difference of FA was independent of these factors. Threshold-free cluster enhancement, ${ }^{21}$ an alternative to conventional cluster-based thresholding, which normally compromises the arbitrary definition of the clusterforming threshold, was used to identify the significant differences between the 2 groups at $P<.05$. Multiple comparisons were accounted for by controlling for the family-wise error rate. Only clusters still significant after the voxel-level family-wise error (part of FSL) correction were reported. The most probable anatomic localization of each cluster was determined by the FSL Atlases tool (http://fsl.fmrib.ox.ac.uk/fsl/fslwiki/Atlases), which incorporates several anatomic templates, including the Talairach atlas, Montreal Neurological Institute structural atlas, Julich histologic atlas, Oxford thalamic connectivity atlas, Harvard-Oxford cortical and subcortical structural atlases, and the Johns Hopkins University DTI-based WM atlases. All reported brain images were acquired by using the "tbss_fill" script from the FSL package.

\section{Volume-of-Interest Analysis of Diffusion Indices}

Volume-of-interest analysis was performed to explore the microstructural mechanisms of the observed changes in FA. Changes in the axial and radial diffusivities (axial, principal longitudinal direction, $\lambda_{1}$, and radial, transverse diffusion component, $\left[\lambda_{\perp}=\right.$ $(\lambda 2+\lambda 3) / 2])$ of clusters showing significant FA decrease were calculated. VOI masks were first extracted on the basis of clusters showing significant intergroup FA differences. These VOI masks were then back-projected to the original images of each subject. The mean values of the diffusion indices within the VOIs were then calculated. A 1-sample Kolmogorov-Smirnov test was performed to evaluate the normality of the data. A 1-way analysis of covariance with the group as the independent variable and diffusion indices as the dependent variables was performed after controlling for age, formal years of education, and history of smoking. Statistical significance was set at $P<.05$. A Bonferroni correction was performed for multiple comparisons. A Pearson correlation analysis was used to assess correlations between changes in FA within the VOIs and behavioral measures and the duration of CCS use. $P<.05$ was considered statistically significant.

\section{RESULTS}

\section{Demographic and Behavioral Measures}

There were no significant differences in age, sex, formal years of education, or number of cigarettes smoked per day between the chronic CCS users and controls. The mean duration of CCS use was 5.05 years. The mean age of the first use of CCS was 19.93 years. The mean dose of CCS used was $487.33 \mathrm{~mL} /$ day in CCS users (Table 1).

A comparison of average of BIS 11 scores from the chronic CCS users and control groups is shown in Table 1. CCS users had significantly higher attentional impulsivity, motor impulsivity, nonplan impulsivity, and total scores than the control group $(P<.05)$.

\section{TBSS}

A value of 0.2 was used to threshold the mean FA skeleton volume so that a total of 140,033 voxels were entered into the voxelwise TBSS analysis. The spatial distribution of the brain regions is presented in Fig 1 and Table 2. Chronic CCS users had lower FA than controls. CCS users had a significantly lower FA $(P<.05$; threshold-free cluster enhancement-corrected $)$ in the inferior fronto-occipital fasciculus (IFO) of the bilateral temporo-occipital regions and the right frontal and the right corona radiata regions than controls. There were no white matter regions in which the controls had significantly lower FA values than the CCS users group. 
VOI

Five clusters showed significant differences between the 2 groups, so the 2 left temporo-occipital IFO clusters were combined into 1 cluster. Four parts of the brain that showed significantly low FA values in CCS users were extracted for VOI-based analysis. The results are listed in Table 3. Clusters in CSS users from the bilateral

temporo-occipital regions of the IFO and the right corona radiata had lower FA values, greater radial diffusivity $(P=.000 ; P \leq .001$, and $P \leq .011$, respectively), and a lower axial diffusivity $(P \leq .027$; $P \leq .014$, and $P \leq .018$, respectively) than clusters from controls. The right frontal part of the IFO had a trend-level increase in radial diffusivity $(P \leq .001)$ and no difference in longitudinal diffusivity $(P=.097)$.

The Pearson correlation analysis demonstrated significant negative correlations between $\mathrm{FA}$ values of the right frontal region of the IFO $(r=-0.479$, $P=.007)$ and the right superior corona radiata WM $(r=-0.450, P=.013)$ with the BIS total scores (Fig 2) of chronic CCS users. There was a significant negative correlation between FA values of the right frontal region of the IFO $(r=$ $-0.479, P=.007)$ and nonplan scores (Fig 2) of chronic CCS users. There was also a significant negative correlation between FA values of the right frontal region of the IFO $(r=-0.561, P=.001)$ and the duration of CCS use (Fig 3 ) in chronic CCS users.

B
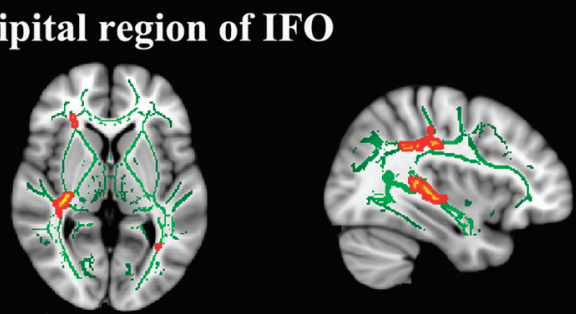

Left temporal-occipital region of IFO

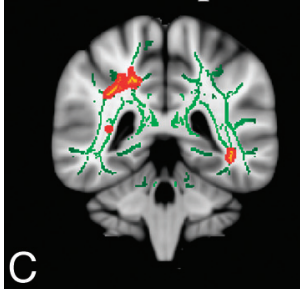

Right superior corona radiata

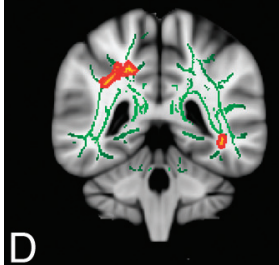

$\mathrm{D}$
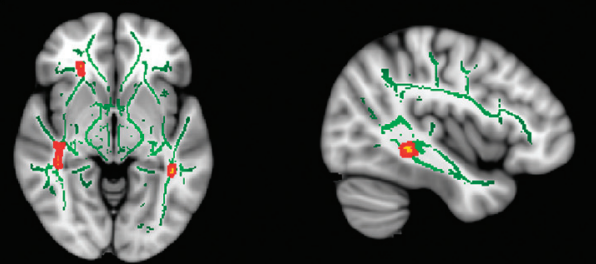

corrected

0.05

FIG 1. TBSS analysis of FA maps. Maps of the $t$ value ( $P<.05$ corrected for multiple comparisons) show, in red, the areas of significantly reduced FA in chronic codeine-containing cough syrup users compared with controls. $A$, The frontal region of the right IFO. $B$, The temporo-occipital region of the right IFO. C, The temporo-occipital region of left IFO. $D$, The right superior corona radiata.
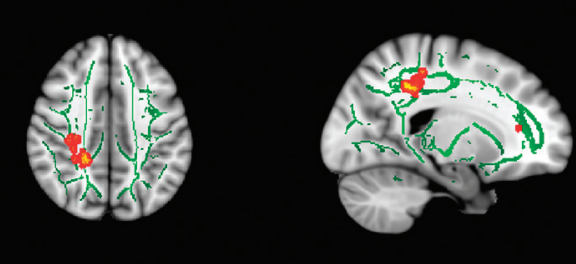

Table 2: Neuroanatomic regions with reduced FA in CCS users relative to healthy controls

\begin{tabular}{|c|c|c|c|c|c|c|}
\hline \multirow[b]{2}{*}{ Anatomic Regions } & \multirow[b]{2}{*}{ Laterality } & \multirow[b]{2}{*}{$\begin{array}{c}\text { Talairach } \\
\text { Coordinates }(x, y, z)^{a}\end{array}$} & \multirow[b]{2}{*}{$\begin{array}{l}\text { Cluster } \\
\text { Size }(\mathrm{cm})\end{array}$} & \multicolumn{2}{|c|}{ Average FA } & \multirow[b]{2}{*}{$\begin{array}{c}P \text { Values } \\
\text { (Minimum) }^{\mathrm{b}}\end{array}$} \\
\hline & & & & $\begin{array}{l}\text { CCS } \\
\text { Users }\end{array}$ & Controls & \\
\hline Frontal region of the IFO & $\mathrm{R}$ & $67,154,68$ & 136 & 0.533 & 0.564 & .048 \\
\hline \multirow[t]{3}{*}{ Temporo-occipital region of IFO } & $\mathrm{R}$ & $54,95,77$ & 389 & 0.452 & 0.499 & .031 \\
\hline & L & $130,84,65$ & 40 & 0.622 & 0.661 & .042 \\
\hline & & $123,66,72$ & 38 & 0.477 & 0.549 & .046 \\
\hline Superior corona radiata & $\mathrm{R}$ & $71,83,112$ & 586 & 0.578 & 0.646 & .031 \\
\hline
\end{tabular}

Note:- L indicates left; R, right.

${ }^{a}$ All the coordinates are denoted by Montreal Neurological Institute space coordinates.

${ }^{\mathrm{b}} P<.05$, threshold-free cluster enhancement-corrected) 
Table 3: Group differences in diffusivity indices from volumes of interest (corrected for age, education, and histories of smoking)

\begin{tabular}{|c|c|c|c|c|c|c|}
\hline \multirow[b]{2}{*}{ Anatomic Region } & \multicolumn{3}{|c|}{$\lambda_{1}\left(\times 10^{-3} \mathrm{~mm}^{2} / \mathrm{s}\right)$ (Mean) } & \multicolumn{3}{|c|}{$\lambda_{\perp}\left(\times 10^{-3} \mathrm{~mm}^{2} / \mathrm{s}\right)$ (Mean) } \\
\hline & Controls & CCS & $P$ Value & Controls & CCS & $P$ Value \\
\hline Right frontal part of IFO & $1.37 \pm 0.05$ & $1.35 \pm 0.05$ & .097 & $0.50 \pm 0.05$ & $0.54 \pm 0.03$ & $.001^{\mathrm{a}}$ \\
\hline Right temporo-occipital part of IFO & $1.43 \pm 0.08$ & $1.37 \pm 0.06$ & $.014^{\mathrm{a}}$ & $0.40 \pm 0.05$ & $0.45 \pm 0.04$ & $.001^{\mathrm{a}}$ \\
\hline Left temporo-occipital part of IFO & $1.44 \pm 0.07$ & $1.40 \pm 0.07$ & $.027^{\mathrm{a}}$ & $0.46 \pm 0.05$ & $0.51 \pm 0.04$ & $.000^{\mathrm{a}}$ \\
\hline Right superior corona radiata & $1.27 \pm 0.07$ & $1.22 \pm 0.07$ & $.018^{\mathrm{a}}$ & $0.53 \pm 0.05$ & $0.56 \pm 0.04$ & $.011^{\mathrm{a}}$ \\
\hline
\end{tabular}

a $p<.05$.

\section{Right frontal region of IFO}
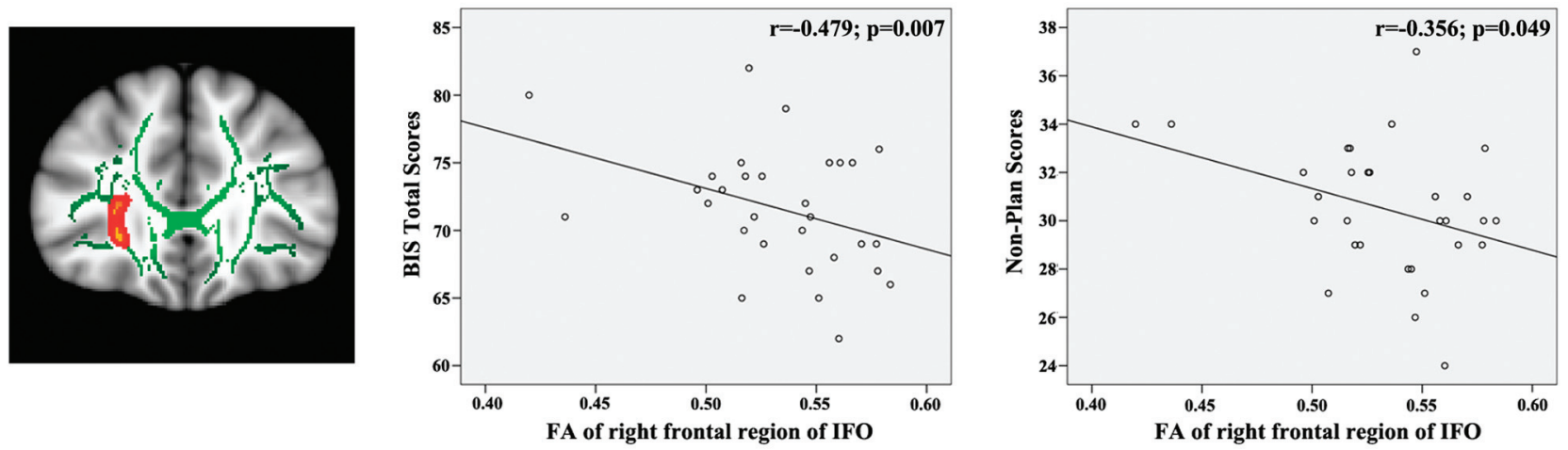

\section{Right superior corona radiata}
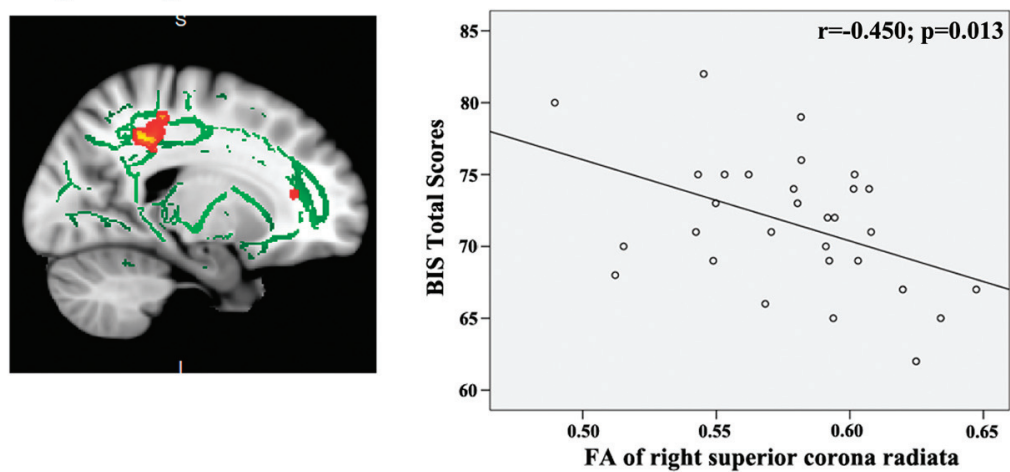

FIG 2. Correlations between mean fractional anisotropy and impulsivity traits in the chronic CCS users.
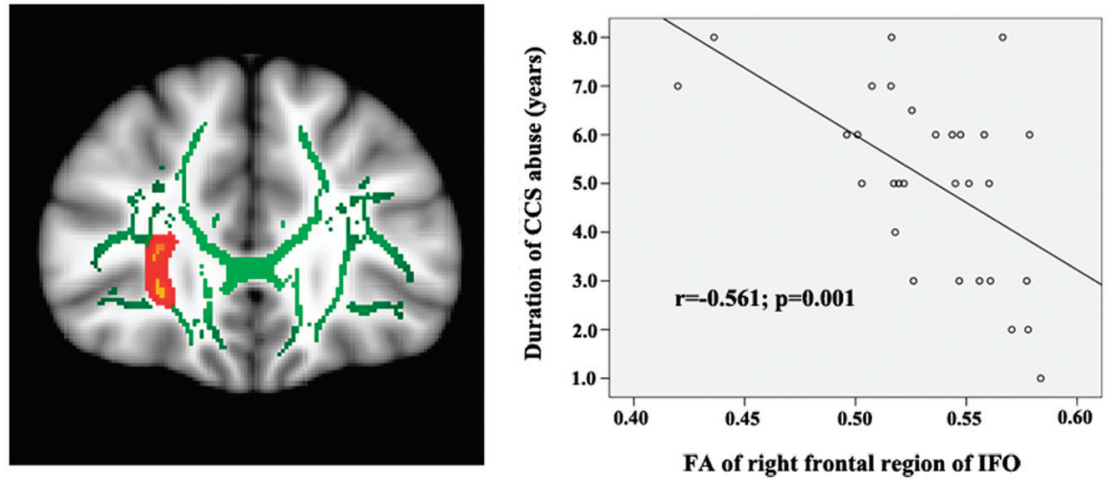

FIG 3. Correlations between mean fractional anisotropy of the right frontal region of the IFO and the duration of CCS use in chronic CCS users.

of the right frontal region of the IFO and the right superior corona radiata WM was negatively correlated with BIS total scores; FA of the right frontal region of the IFO was negatively correlated with nonplan impulsivity and the duration of CCS use in chronic CCS users (Fig 2). These findings suggest that chronic CCS abuse may cause progressive brain WM integrity impairment. Furthermore, these WM microstructural deficits may be linked to higher impulsivity traits in chronic CCS users.

\section{Abnormal WM Integrity in Chronic CCS Users}

A low FA value was found in the bilateral IFO, the right frontal region, and the bilateral temporo-occipital regions of chronic CSS users. The IFO is thought to be part of the dorsal visual stream, ${ }^{22}$ originating from the dorsal parieto-occipital and medial parietal areas and projecting into the lateral prefrontal areas. ${ }^{23}$ The IFO is thought to be involved in processing information that controls action. ${ }^{24}$ Abnormal WM integrity in the IFO has consistently been observed in other forms of addiction, such as Internet, ${ }^{25}$ 
alcohol, ${ }^{26}$ and heroin. ${ }^{27}$ The observation of a lower FA value in the bilateral IFO of chronic CCS users is consistent with these previous reports and with the clinical observation that CCS abuse is associated with impaired cognitive control. ${ }^{2-4}$ The anterior part of the IFO may be involved in response inhibition. Increased coherence of the anterior part of the IFO might give rise to an increased ability to implement inhibition to overcome the incorrect visuospatial task preparation and hence the incorrect competing response found in invalidly but not validly cued trials. ${ }^{28}$ The negative correlation among FA values of the right frontal region of the IFO and BIS total scores and nonplan scores supports this hypothesis.

The right superior corona radiata WM also had a lower FA value in CCS users. The corona radiata comprises WM fibers linking the cerebral cortex to the internal capsule and provides important connections among the frontal, parietal, temporal, and occipital lobes. ${ }^{29}$ Given its anatomic location, the corona radiata WM has been linked to risk-taking, including both cognitive and motor components. ${ }^{29,30}$ The abnormal WM integrity of the right superior corona radiata observed in this study may account for the risk-taking behavior and higher impulsivity found in chronic CCS users. Loss of WM integrity in the corona radiata has been consistently observed in cocaine, ${ }^{31}$ methamphetamine, ${ }^{32}$ and alcohol dependence. ${ }^{33}$ These findings are consistent with those of previous results. The significant negative correlation between the FA value of the right superior corona radiata and the BIS total score supports the conclusions of previous functional studies linking the parietal lobe to impulse control. ${ }^{34}$

Results also showed the FA values of the right frontal region of the IFO to be negatively correlated to the duration of CCS use. The disruption of white matter integrity in this region may be due to this use. The FA values of the 3 other parts of the brain regions that showed differences between users and controls did not demonstrate any such correlation. Several factors might explain these findings. First, the relatively small sample size could have insufficient power to detect such a difference. Although no significant correlation was found between the FA values of the other 3 parts of the brain and the duration of CCS use, negative trends were observed. Further studies with larger sample sizes are needed. Second, the lower FA values in these regions may not be the result of CCS abuse. They may represent a predisposition to substance abuse. ${ }^{35}$ Further studies with long-term follow-up are needed to confirm this inference.

\section{Possible Mechanisms Underlying Decreases in FA}

The FA value of WM may be affected by many factors, including myelination, axon size, axon attenuation, axonal path geometry, and extracellular water space between fibers. ${ }^{36}$ Results showed CCS-related WM microstructural damage to be associated with reduced axial diffusivity and increased radial diffusivity in bilateral temporo-occipital regions of the IFO and right superior corona radiata WM. Radial diffusivity mainly reflects the integrity and thickness of myelin sheets covering the axons. ${ }^{12}$ Axial diffusivity may reflect the organization of the fibers and axon integrity. ${ }^{12}$ The disruption of WM integrity seen in the brain of chronic CCS users is most likely a manifestation of the loss of myelin and axon integrity. Postmortem and DTI studies have also demon- strated myelin dissolution ${ }^{28}$ and axon disruption ${ }^{37}$ in opiate-dependent individuals. Büttner et $\mathrm{al}^{37}$ found $\beta$-amyloid precursor protein-immunopositive accumulations to be significantly more pronounced in heroin-predominant polydrug addicts, indicating diffuse axonal damage. Similarly, Bora et $\mathrm{al}^{27}$ demonstrated widespread deficits of white matter integrity in heroin addicts.

\section{Relationship between FA and Behavioral Measures in Chronic CCS Users}

Chronic CCS users showed significantly higher BIS total scores, attentional scores, nonplan scores, and motor scores than controls. These findings are consistent with the results of previous neuropsychological studies of heroin and cocaine addicts. ${ }^{38}$ Results also showed that the BIS total scores were correlated to the FA values of the right frontal region of the IFO and the right superior corona radiata in CCS users. Higher nonplan impulsivity scores were associated with more severe loss of white matter integrity in the right frontal region of the IFO. This association indicates that disruption of the WM integrity in these regions may be responsible for the higher impulsivity found in chronic CCS users. These inferences are supported by a previous study by Lane et $\mathrm{al}^{39}{ }^{39}$ which showed a significant relationship between impaired decision-making (by Iowa gambling task) and DTI-measured white matter integrity in the frontal and parietal regions of cocaine-dependent subjects. In turn, the findings of significant associations among impaired white matter integrity in the right frontal region of the IFO and the right superior corona radiata WM and higher impulsivity in CCS users suggest that white matter integrity may serve as a predictor of abstinence or a potential new treatment target for CCS users.

\section{Limitations of This Study}

There were several limitations to the current study. First, the study was cross-sectional in design. The integrity of abnormal white matter was observed in CCS users, and associations with chronic CCS use were inferred. However, we cannot absolutely assert the causation of the CCS dependence and the abnormalities of the white matter integrity. It requires a long-term longitudinal follow-up with detailed experimental and clinical profiling of a cohort of subjects in a future study. The second limitation of this study concerns imaging. The assessment of small fiber tracts was difficult with TBSS because only the major white matter tracts were included in the skeleton. ${ }^{13}$ Because of the nonlinear alignment process, the FA, $\lambda_{\perp}$, and $\lambda_{1}$ values that were attributed to a given voxel were not actually measured from that spatial location. Instead, they were derived from a neighborhood, which may disguise the results. ${ }^{13}$ Third, the DTI sequence had only 1 acquisition at $b=0 \mathrm{~s} / \mathrm{mm}^{2}$, due to limitations of the imager. ${ }^{40}$ Last, the lifetime quantity of alcohol use was not measured in present study; although none of the subjects included in present study met the Diagnostic and Statistical Manual of Mental Disorders-IV criteria for alcohol dependence, binge drinking has been found to be associated with reduced FA in previous studies. ${ }^{41}$ Heavier alcohol use without dependence could be related to the present findings.

\section{CONCLUSIONS}

DTI was used with TBSS analysis to investigate the microstructure of WM in chronic CCS users. Chronic CCS users are character- 
ized by impairment of WM fibers in the bilateral temporo-occipital regions and the right frontal region of the IFO and the right superior corona radiata. In addition, these progressive changes in WM microstructure may be neural and cause greater impulsivity in chronic CCS users.

\section{REFERENCES}

1. Hutchings HA, Eccles R. The opioid agonist codeine and antagonist naltrexone do not affect voluntary suppression of capsaicin induced cough in healthy subjects. Eur Respir J 1994;7:715-19

2. Vree TB, van Dongen RT, Koopman-Kimenai PM. Codeine analgesia is due to codeine-6-glucuronide, not morphine. Int J Clin Pract 2000;54:395-98

3. Shek DT, Lam CM. Adolescent cough medicine abuse in Hong Kong: implications for the design of positive youth development programs in Hong Kong. Int J Adolesc Med Health 2006;18:493-503

4. Mattoo SK, Basu D, Sharma A, et al. Abuse of codeine-containing cough syrups: a report from India. Addiction 1997;92:1783-87

5. Hou H, Yin S, Jia S, et al. Decreased striatal dopamine transporters in codeine-containing cough syrup abusers. Drug Alcohol Depend 2011;118:148-51

6. Qiu Y, Lv X, Su H, et al. Reduced regional homogeneity in bilateral frontostriatal system relates to higher impulsivity behavior in codeine-containing cough syrups dependent individuals. PLoS One 2013;8:e78738

7. Qiu Y, Lv X, Jiang G, et al. Reduced ventral medial prefrontal cortex (vmPFC) volume and impaired vmPFC-default mode network integration in codeine-containing cough syrups users. Drug Alcohol Depend 2014;134:314-21

8. Le Bihan D, Mangin JF, Poupon C, et al. Diffusion tensor imaging: concepts and applications. J Magn Reson Imaging 2001;13:534-46

9. Kubicki M, Westin CF, Maier SE, et al. Diffusion tensor imaging and its application to neuropsychiatric disorders. Harv Rev Psychiatry 2002;10:324-36

10. Wozniak JR, Lim KO. Advances in white matter imaging: a review of in vivo magnetic resonance methodologies and their applicability to the study of development and aging. Neurosci Biobehav Rev 2006;30:762-74

11. Seal ML, Yücel M, Fornito A, et al. Abnormal white matter microstructure in schizophrenia: a voxelwise analysis of axial and radial diffusivity. Schizophr Res 2008;101:106-10

12. Song SK, Yoshino J, Le TQ, et al. Demyelination increases radial diffusivity in corpus callosum of mouse brain. Neuroimage 2005;26:132-40

13. Smith SM, Jenkinson M, Johansen-Berg H, et al. Tract-based spatial statistics: voxelwise analysis of multi-subject diffusion data. Neuroimage 2006;31:1487-505

14. Patton JH, Stanford MS, Barratt ES. Factor structure of the Barratt impulsiveness scale. J Clin Psychol 1995;51:768-74

15. Yao S, Yang H, Zhu X, et al. An examination of the psychometric properties of the Chinese version of the Barratt Impulsiveness Scale, 11th version in a sample of Chinese adolescents. Percept Mot Skills 2007;104:1169-82

16. Smith S, Jenkinson M, Woolrich $M$, et al. Advances in functional and structural MR image analysis and implementation as FSL. Neuroimage 2004;23:208-19

17. Smith $\mathrm{S}$. Fast robust automated brain extraction. Hum Brain Mapp 2002;17:143-55

18. Rueckert D, Sonoda LI, Hayes C, et al. Nonrigid registration using free-form deformations: application to breast MR images. IEEE Trans Med Imaging 1999;18:712-21

19. Versace A, Almeida J, Hassel S, et al. Elevated left and reduced right orbitomedial prefrontal fractional anisotropy in adults with bipo- lar disorder revealed by tract-based spatial statistics. Arch Gen Psychiatry 2008;65:1041-52

20. Jones DK, Symms MR, Cercignani M, et al. The effect of filter size on VBM analyses of DT-MRI data. Neuroimage 2005;26:546-54

21. Smith SM. Threshold-free cluster enhancement: addressing problems of smoothing, threshold dependence and localization in cluster inference. Neuroimage 2009;44:83-98

22. Mishkin M, Ungerleider LG. Contribution of striate inputs to the visuospatial functions of parieto-preoccipital cortex in monkeys. Behav Brain Res 1982;6:57-77

23. Schmahmann JD, Pandya DN, Wang R, et al. Association fibre pathways of the brain: parallel observations from diffusion spectrum imaging and autoradiography. Brain 2007;130:630-53

24. Rizzolatti G, Matelli M. Two different streams form the dorsal visual system: anatomy and functions. Exp Brain Res 2003;153:146-57

25. Lin F, Zhou Y, Du Y, et al. Abnormal white matter integrity in adolescents with internet addiction disorder: a tract-based spatial statistics study. PLoS One 2012;7:e30253

26. Herting MM, Schwartz D, Mitchell SH, et al. Delay discounting behavior and white matter microstructure abnormalities in youth with a family history of alcoholism. Alcohol Clin Exp Res 2010;34:1590-602

27. Bora E, Yücel M, Fornito A, et al. White matter microstructure in opiate addiction. Addict Biol 2012;17:141-8

28. Forstmann BU, Jahfari S, Scholte HS, et al. Function and structure of the right inferior frontal cortex predict individual differences in response inhibition: a model-based approach. J Neurosci 2008;28:9790-96

29. Wakana S. Fiber tract-based atlas of human white matter anatomy. Radiology 2004;230:77-87

30. Berns GS, Moore S, Capra CM. Adolescent engagement in dangerous behaviors is associated with increased white matter maturity of frontal cortex. PLoS One 2009;4:e6773

31. Bell RP, Foxe JJ, Nierenberg J, et al. Assessing white matter integrity as a function of abstinence duration in former cocaine-dependent individuals. Drug Alcohol Depend 2011;114:159-68

32. Tobias MC, O’Neill J, Hudkins M, et al. White-matter abnormalities in brain during early abstinence from methamphetamine abuse. Psychopharmacology 2010;209:13-24

33. Yeh PH, Simpson K, Durazzo TC, et al. Tract-based spatial statistics (TBSS) of diffusion tensor imaging data in alcohol dependence: abnormalities of the motivational neurocircuitry. Psychiatry Res 2009; 173:22-30

34. Hinvest NS, Elliott R, McKie S, et al. Neural correlates of choice behavior related to impulsivity and venturesomeness. Neuropsychologia 2011;49:2311-20

35. Haney-Caron E, Caprihan A, Stevens MC. DTI-measured white matter abnormalities in adolescents with conduct disorder. J Psychiatr Res 2014;48:111-20

36. Le Bihan D. Looking into the functional architecture of the brain with diffusion MRI. Nat Rev Neurosci 2003;4:469-80

37. Büttner A, Rohrmoser K, Mall G, et al. Widespread axonal damage in the brain of drug abusers as evidenced by accumulation of betaamyloid precursor protein (beta-APP): an immunohistochemical investigation. Addiction 2006;101:1339-46

38. Verdejo-García AJ, Perales JC, Pérez-García M. Cognitive impulsivity in cocaine and heroin polysubstance abusers. Addict Behav 2007;32:950-66

39. Lane SD, Steinberg JL, Ma L, et al. Diffusion tensor imaging and decision making in cocaine dependence. PLoS One 2010;5:e11591

40. Smith S, Johansen-Berg H, Jenkinson M, et al. Acquisition and voxelwise analysis of multi-subject diffusion data with tract-based spatial statistics. Nat Protoc 2007;2:499-503

41. Kong LM, Zheng WB, Lian GP, et al. Acute effects of alcohol on the human brain: diffusion tensor imaging study. AJNR Am JNeuroradiol 2012;33:928-34 